\title{
Aminoguanidina reduz o estresse oxidativo e as alterações estruturais pulmonares em diabetes mellitus experimental*
}

\author{
Aminoguanidine reduces oxidative stress and structural \\ lung changes in experimental diabetes mellitus
}

\author{
Fabio Cangeri Di Naso, Luiz Alberto Forgiarini Junior, Luiz Felipe Forgiarini, \\ Marilene Porawski, Alexandre Simões Dias, Norma Anair Possa Marroni
}

\begin{abstract}
Resumo
Avaliamos o efeito da aminoguanidina sobre o estresse oxidativo pulmonar e a estrutura pulmonar em um modelo experimental de diabetes mellitus. Foram determinados thiobarbituric acid reactive substances (TBARS, substâncias reativas ao ácido tiobarbitúrico), histologia e gasometria arterial em animais com diabetes mellitus (DM), animais com diabetes mellitus tratados com aminoguanidina ( $D M+A G)$ e controles. 0 nível de TBARS foi significativamente maior no grupo DM que nos grupos controle e $\mathrm{DM}+\mathrm{AG}(2,90 \pm 1,12$ vs. 1,62 $\pm 0,28$ e 1,68 $\pm 0,04 \mathrm{nmol} /$ mg proteína, respectivamente), o mesmo ocorrendo com $\mathrm{PaCO}_{2}$ em relação ao grupo controle $(49,2 \pm 1,65$ vs. $38,12 \pm 4,85 \mathrm{mmHg})$, e $\mathrm{PaO}_{2}$ foi significativamente maior no grupo controle $(104,5 \pm 6,3$ vs. 69,48 $\pm 16,30$ e $97,05 \pm 14,02 \mathrm{mmHg}$, respectivamente). Neste modelo experimental de diabetes mellitus, a aminoguanidina reduziu o estresse oxidativo, alterações estruturais teciduais pulmonares e a troca gasosa no modelo experimental.
\end{abstract}

Descritores: Estresse oxidativo; Diabetes mellitus experimental; Pulmão.

\begin{abstract}
We evaluated the effect of aminoguanidine on pulmonary oxidative stress and lung structure in an experimental model of diabetes mellitus. Thiobarbituric acid reactive substances (TBARS), histology and arterial blood gases were evaluated in animals with diabetes mellitus (DM group), animals with diabetes mellitus treated with aminoguanidine (DM+AG group), and controls. The TBARS levels were significantly higher in the DM group than in the control and $\mathrm{DM}+\mathrm{AG}$ groups $\left(2.90 \pm 1.12\right.$ vs. $1.62 \pm 0.28$ and $1.68 \pm 0.04 \mathrm{nmol} / \mathrm{mg}$ protein, respectively), as was $\mathrm{PaCO}_{2}$ when compared with that of the control group ( $49.2 \pm 1.65$ vs. $38.12 \pm 4.85 \mathrm{mmHg})$, and $\mathrm{PaO}_{2}$ was significantly higher in the control group $(104.5 \pm 6.3$ vs. $16.30 \pm 69.48$ and $97.05 \pm 14.02 \mathrm{mmHg}$, respectively). In this experimental model of diabetes mellitus, aminoguanidine reduced oxidative stress, structural tissue alterations, and gas exchange.
\end{abstract}

Keywords: Oxidative stress; Diabetes mellitus, experimental; lung.

0 diabetes mellitus (DM) é um distúrbio metabólico que acomete diversos órgãos. Estima-se que 171 milhões de pessoas estejam afetadas pela doença. Esse número deverá subir para 366 milhões até o ano de 2030, e os fatores principais que interferem no desenvolvimento da doença são o aumento da idade da população, o maior número de indivíduos que desenvolvem a obesidade e a maior urbanização das cidades. Atualmente, o DM ocupa o quinto lugar nas causas de morte no mundo. ${ }^{(1)}$

Diversos estudos clínicos e experimentais têm identificado vários mecanismos fisiopatológicos implicados no desenvolvimento das alterações pulmonares induzidas pelo DM, e parece que a inflamação aguda é um dos principais desencadeadores desse processo. ${ }^{(2,3)}$ Distúrbios na produção de surfactante e dificuldade na absorção do edema também estão presentes, bem como o desequilíbrio nas atividades oxidante/antioxidante, coagulação/fibrinólise e no sistema de fibrose/reparação.

Os principais fatores associados às complicações pulmonares crônicas são o estresse oxidativo e a geração de advanced glycation end products (AGEs, produtos finais de glicação

\footnotetext{
* Trabalho realizado no Hospital de Clínicas de Porto Alegre, Faculdade de Medicina, Universidade Federal do Rio Grande do Sul UFRGS - Porto Alegre (RS) Brasil.

Endereço para correspondência: Norma Marroni. Rua José Kanan Aranha, 102, Jardim 1sabel, CEP 91760-470, Porto Alegre, RS, Brasil.

Tel 5551 3269-0663. Email: nmarroni@terra.com.br

Apoio financeiro: Este estudo recebeu apoio financeiro do Fundo de Incentivo a Pesquisa (FIPE), Hospital de Clinicas de Porto Alegre (HCPA). Luiz Felipe Forgiarini é bolsista de iniciação científica da instituição.

Recebido para publicação em 17/3/2010. Aprovado, após revisão, em 22/4/2010.
} 
avançada). ${ }^{(4)}$ Nosso grupo já demonstrara em um estudo que o estresse oxidativo está presente no DM experimental, e os animais que apresentavam a doença possuíam alterações estruturais no tecido pulmonar, bem como mudança nos valores dos gases sanguíneos. ${ }^{(5)}$

A utilização de uma terapêutica que bloqueie os AGEs ou reduza o estresse oxidativo e nitrosativo pode ser eficaz na doença pulmonar.

A aminoguanidina previne a formação das AGEs porque contém hidrazina em sua estrutura química, a qual reage com os seguintes compostos: glioxal, metilglioxal e 3-desoxiglicosona. ${ }^{(6)}$ Além dos efeitos sobre a glicação avançada, a aminoguanidina em doses mais baixas pode atuar de maneira específica inibindo a atividade da enzima iNOS e reduzir 0 estresse nitrosativo. ${ }^{(7)}$ A aminoguanidina também pode inibir o metabolismo da histamina, o catabolismo de poliaminas e a atividade da catalase, assim como potencializar os efeitos da angiotensina na produção de prostaciclina. No entanto, nenhum estudo experimental demonstrou os efeitos da aminoguanidina sobre as complicações pulmonares decorrentes do DM. ${ }^{(8)}$

Este estudo teve como objetivo avaliar o efeito da aminoguanidina na redução do estresse oxidativo e dos possíveis danos existentes na estrutura pulmonar provocados pelo DM. Foram realizadas medidas gasométricas sanguíneas para avaliar as alterações existentes na troca gasosa, assim como foi feita a avaliação do dano oxidativo tecidual com o uso de técnicas histológicas para determinar e quantificar as alterações existentes na estrutura pulmonar.

Este é um estudo experimental controlado, no qual foram utilizados ratos da raça Wistar pesando, em média, $300 \mathrm{~g}$. Todos os animais foram tratados conforme o Ethical Code for Animal Experimentation preconizado pela Organização Mundial da Saúde. Os animais foram divididos em três grupos: grupo controle, grupo de animais com DM e grupo de animais com DM que foram tratados com aminoguanidina. Cada grupo foi composto por sete animais. 0 período do estudo foi de 60 dias, a contar da data em que os animais diabéticos apresentaram glicemia sanguínea superior a $250 \mathrm{mg} / \mathrm{dL}$.

0 DM foi induzido com uma única injeção i.p. de estreptozotocina (Sigma Chemical, St. Louis, $\mathrm{MO}, \mathrm{EUA})$ na dose de $70 \mathrm{mg} / \mathrm{kg}$ de peso corporal.
(9) Para os ratos tratados com aminoguanidina (aminoguanidine hemisulfate salt, SigmaAldrich, St. Louis, MO, EUA), foi utilizada a dose i.p. de $50 \mathrm{mg} / \mathrm{kg}$ de peso durante os últimos 30 dias do experimento. ${ }^{(10)}$

Para a determinação da glicemia, utilizou-se o teste enzimático colorimétrico, e os animais foram sacrificados no $60^{\circ}$ dia de experimento. Todos foram anestesiados (cetamina, $100 \mathrm{mg} / \mathrm{kg}$ + xilasina, $50 \mathrm{mg} / \mathrm{kg}$; i.p.) e em seguida submetidos a tricotomia e laparotomia ventral media na região torácica e abdominal. Foi coletado sangue da artéria aorta abdominal no intuito de avaliar os gases arteriais. Foi utilizado um analisador ABL 700 (Radiometer, Copenhagen, Dinamarca) para a determinação de $\mathrm{PaO}_{2}, \mathrm{PaCO}_{2}$ e $\mathrm{SaO}_{2}$. Posteriormente, os pulmões foram retirados e fixados em paraformaldeído a 4\% para a análise histológica, sendo uma parte armazenada em freezer na temperatura de $-80^{\circ} \mathrm{C}$ para a posterior quantificação de thiobarbituric acid reactive substances (TBARS, substâncias reativas ao ácido tiobarbitúrico). A medida das TBARS foi realizada conforme o estabelecido por Buege e Aust. ${ }^{(11)}$

Para a realização da peroxidação lipídica, o tecido pulmonar foi homogeneizado ${ }^{(12)} \mathrm{e}$, em seguida, foi realizada a quantificação da proteína segundo o método de Lowry et al. ${ }^{(13)}$

Para a análise histológica pulmonar, foi utilizada a técnica com picrosírius red. 0 exame anatomopatológico foi realizado de modo duplo cego por um patologista do Laboratório de Patologia do Hospital das Clínicas de Porto Alegre. Os dados foram analisados no programa Statistical Package for the Social Sciences versão 13 (SPSS Inc., Chicago, IL, EUA), sendo utilizado o teste de ANOVA para a comparação entre os grupos e, como post hoc, o teste de StudentNewman-Keuls para as comparações entre os grupos. 0 nível de significância adotado foi de $5 \%(p<0,05)$.

Quando analisada a concentração de glicose sanguínea, verificou-se um aumento significativo no grupo diabético quando comparado ao grupo controle e, após o tratamento com aminoguanidina, não houve redução da hiperglicemia. A peroxidação lipídica pulmonar foi maior nos animais diabéticos quando comparados aos do grupo controle, e a utilização da aminoguanidina reduziu significativamente os níveis de peroxidação lipídica tecidual, 
Tabela 1 - Comparação dos resultados de glicemia, substâncias reativas ao ácido tiobarbitúrico e gases sanguíneos entre os grupos.

\begin{tabular}{|c|c|c|c|}
\hline \multirow[t]{3}{*}{ Parâmetros } & \multicolumn{3}{|c|}{ Grupo } \\
\hline & Controle & DM & $\mathrm{DM}+\mathrm{AG}$ \\
\hline & $(n=7)$ & $(n=7)$ & $(n=7)$ \\
\hline Glicemia, mg/dL & $217,6 \pm 11,84$ & $561 \pm 35,60^{*}$ & $549,62 \pm 42,30^{* *}$ \\
\hline TBARS, nmol/mg proteína & $1,62 \pm 0,28$ & $2,90 \pm 1,12^{*}$ & $1,68 \pm 0,40^{* * *}$ \\
\hline $\mathrm{PaO}_{2}, \mathrm{mmHg}$ & $104,58 \pm 6,33$ & $69,48 \pm 16,30^{*}$ & $97,05 \pm 14,02^{* * * *}$ \\
\hline $\mathrm{PaCO}_{2}, \mathrm{mmHg}$ & $38,12 \pm 4,85$ & $49,2 \pm 1,65^{*}$ & $45,28 \pm 10,84$ \\
\hline $\mathrm{SaO}, \%$ & $97,54 \pm 0,55$ & $84,92 \pm 7,80^{*}$ & $94,6 \pm 3,24^{* * *}$ \\
\hline
\end{tabular}

DM: animais com diabetes mellitus; DM+AG: animais com diabetes mellitus tratados com aminoguanidina; e TBARS: thiobarbituric acid reactive substances (substâncias reativas ao ácido tiobarbitúrico). Valores expressos em média $\pm \mathrm{dp}$. ${ }^{*} p<0,05$ (grupo diabético vs. grupo controle). ${ }^{* *} p<0,05$ (grupo diabético tratado com AG vs. grupo controle). ${ }^{* * *} p<0,05$ (grupo diabético tratado com AG vs. grupo diabético).

quase retornando aos valores basais. $\mathrm{Na}$ análise gasométrica, observamos um aumento em $\mathrm{PaCO}_{2}$ no grupo diabético, bem como uma redução em $\mathrm{PaO}_{2}$ e $\mathrm{SaO}_{2}$, quando comparados aos animais do grupo controle. Entretanto, ao analisarmos os gases sanguíneos dos animais diabéticos tratados com aminoguanidina, houve um aumento significativo em $\mathrm{PaO}_{2}$ e $\mathrm{SaO}_{2}$ (Tabela 1).

$\mathrm{Na}$ analise histológica, evidenciou-se a presença de células inflamatórias no grupo diabético, quando comparado ao grupo controle, e também no grupo diabético que recebeu aminoguanidina. No grupo diabético também houve um aumento na matriz extracelular, a qual determina a presença de fibrose tecidual. Quando quantificada a fibrose, houve um aumento significativo dessa no grupo diabético quando comparada a do grupo controle, assim como uma redução estatisticamente significativa nos animais diabéticos tratados com aminoguanidina (Figura 1).
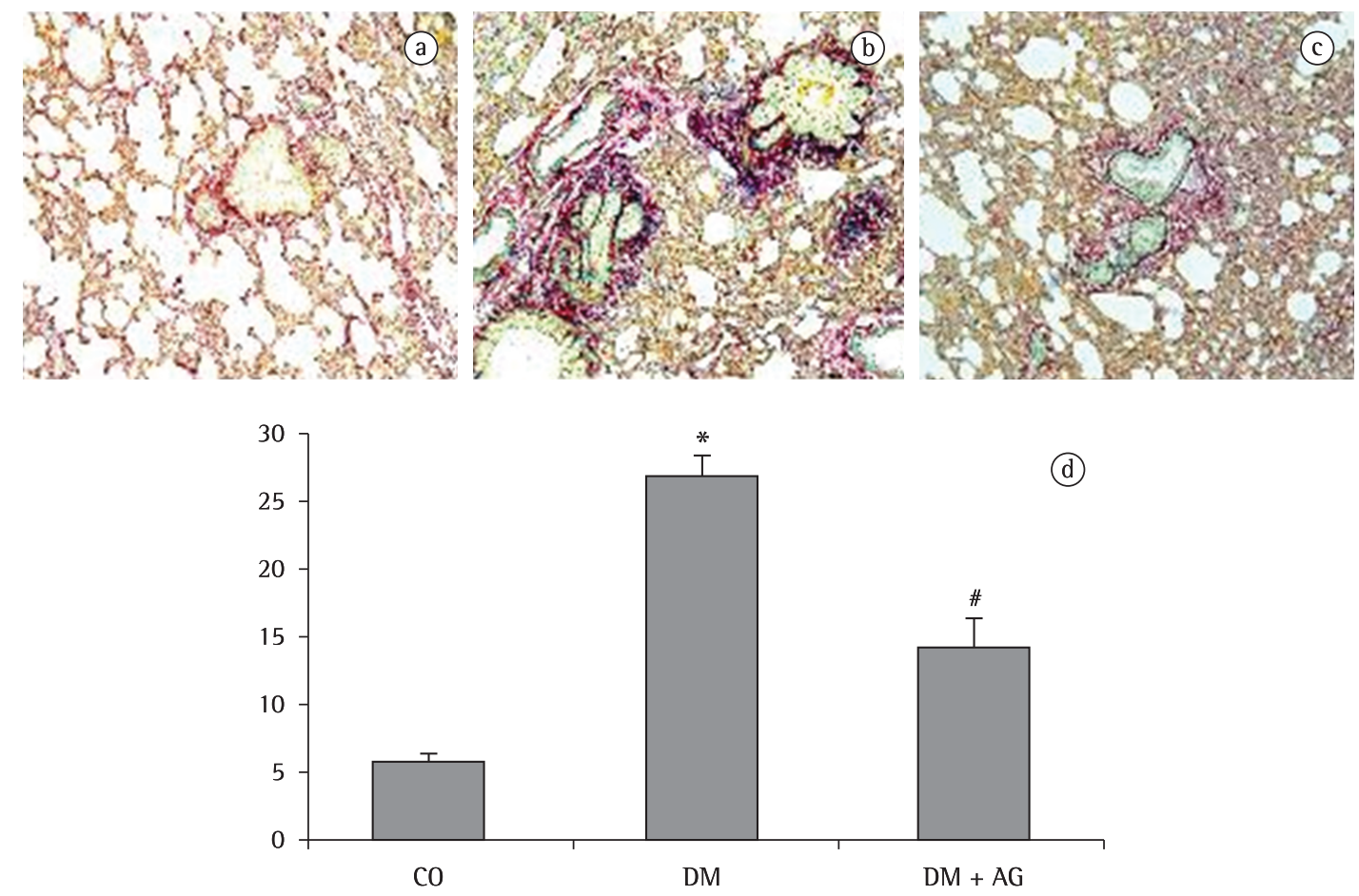

Figura 1 - Fotos de microscopia das amostras de tecido pulmonar de animais dos grupos: em a), controle (CO); em b), diabetes mellitus (DM); e em c), diabetes mellitus tratado com aminoguanidina (DM+AG; picrosirius red; aumento, 200×). Em d), gráfico da quantificação da fibrose (percentual de pixels). ${ }^{*} p<0,001$ vs. grupos CO e $\mathrm{DM}+\mathrm{AG} ;{ }^{*} \mathrm{p}<0,001$ vs. grupo DM. 
Neste estudo, assim como em um estudo realizado anteriormente, ${ }^{(5)}$ observamos um aumento na peroxidação lipídica do tecido pulmonar em animais diabéticos. No presente estudo, a aminoguanidina reduziu a peroxidação lipídica. Apesar de ser uma medida indireta na avaliação do estresse oxidativo, a análise da peroxidação lipídica pode indicar dano celular mediado por agentes oxidantes e nitrosantes. Estudos prévios demonstraram a capacidade da aminoguanidina em reduzir a apoptose induzida pelas espécies reativas de oxigênio e pela peroxidação lipídica em células da retina e de rim em animais com DM. ${ }^{(14,15)}$

$\mathrm{Na}$ gasometria realizada, encontramos alterações na troca gasosa nos animais diabéticos, pois houve diminuição em $\mathrm{PaO}_{2}$ e $\mathrm{SO}_{2}$ e aumento em $\mathrm{PaCO}_{2}$ sanguínea. Um resultado semelhante foi encontrado em pacientes diabéticos, pois também há uma redução significativa na capacidade de difusão pulmonar quando comparada a de indivíduos hígidos. Um dos prováveis fatores preditores para essa alteração clínica foi a microalbuminuria apresentada pelos pacientes, pois a mesma demonstrou uma correlação inversa com a difusão pulmonar. ${ }^{(16)}$

Um dos fatores responsáveis por essa alteração no sistema pulmonar pode ter sido o aumento na espessura da membrana basal, já que a mesma pode ser ocasionada pelas ligações inter e intramoleculares com o colágeno, que são o resultado da ação dos AGEs. Esse mecanismo também pode contribuir para aumentar a rigidez e a resistência à digestão proteolítica, bem como interferir nas proteínas da matriz extracelular (fibronectina; colágeno tipo III, IV e Vl; e laminina) e causar upregulation na formação de citocinas. ${ }^{(17)}$

A utilização da aminoguanidina demonstrou ser eficaz no modelo experimental de nefropatia diabética, pois houve redução no espessamento da membrana basal glomerular após sua utilização. ${ }^{(15)}$ No presente estudo, a aminoguanidina melhorou a capacidade difusional dos gases sanguíneos, pois houve aumento em $\mathrm{PaO}_{2}$ e $\mathrm{SaO}_{2}$. No entanto, não houve redução de $\mathrm{PaCO}_{2}$ após o tratamento. Isso pode ser explicado pelas prováveis alterações existentes na mecânica ventilatória e na ação dos quimiorreceptores centrais e periféricos que também estão afetados no DM. . $^{(5,18)}$
Quando analisamos a histologia do tecido pulmonar, evidenciamos um aumento na barreira alveolocapilar nos animais diabéticos. Essas alterações também foram observadas por alguns autores, ${ }^{(18,19)}$ os quais demonstraram que o DM é o principal responsável por alterações estruturais pulmonares. Segundo um estudo no qual se avaliou a inibição de nuclear factor kappa $B$ (NF-кB, fator de transcrição nuclear kappa B) em modelo experimental de DM, foi demonstrado que o pulmão de animais diabéticos apresenta alterações na estrutura tecidual, e que essas foram ocasionadas pelo aumento do estresse oxidativo, e, quando NF- $\mathrm{KB}$ foi inibido, houve redução na injúria pulmonar induzida pela doença. ${ }^{(20)}$

A aminoguanidina possivelmente atua como um inibidor dos AGEs, pois reduz os níveis de estresse oxidativo pulmonar e diminui as alterações estruturais pulmonares causadas pelo aumento na síntese e na deposição de colágeno.

Em resumo, alterações estruturais no tecido pulmonar de animais diabéticos ocasionam modificações nas trocas gasosas, bem como um aumento do estresse oxidativo. Após a utilização da aminoguanidina, observou-se uma redução na peroxidação lipídica e nas alterações estruturais pulmonares expressas através da fibrose, bem como o restabelecimento dos níveis de $\mathrm{PaO}_{2}$ e $\mathrm{SaO}_{2}$.

\section{Referências}

1. Diabetes Prevention Program Research Group; Knowler WC, Fowler SE, Hamman RF, Christophi CA, Hoffman $\mathrm{HJ}$, et al. 10-year follow-up of diabetes incidence and weight loss in the Diabetes Prevention Program Outcomes Study. Lancet. 2009;374(9702):1677-86. Erratum in: Lancet. 2009;374(9707):2054.

2. Gumieniczek A, Wilk M. Nitrosative stress and glutathione redox system in four different tissues of alloxan-induced hyperglycemic animals. Toxicol Mech Methods. 2009;19(4):302-7.

3. Foster DJ, Ravikumar P, Bellotto DJ, Unger RH, Hsia CC. Fatty diabetic lung: altered alveolar structure and surfactant protein expression. Am J Physiol Lung Cell Mol Physiol. 2010;298(3):L392-403.

4. Honiden S, Gong MN. Diabetes, insulin, and development of acute lung injury. Crit Care Med. 2009;37(8):245564.

5. Forgiarini LA Jr, Kretzmann NA, Porawski M, Dias AS, Marroni NA. Experimental diabetes mellitus: oxidative stress and changes in lung structure. J Bras Pneumol. 2009;35(8):788-91.

6. Thornalley PJ. Use of aminoguanidine (Pimagedine) to prevent the formation of advanced glycation 
endproducts. Arch Biochem Biophys. 2003;419(1):3140.

7. Szabó C, Ferrer-Sueta G, Zingarelli B, Southan GJ, Salzman AL, Radi R Mercaptoethylguanidine and guanidine inhibitors of nitric-oxide synthase react with peroxynitrite and protect against peroxynitrite-induced oxidative damage. J Biol Chem. 1997;272(14):9030-6.

8. Nilsson B0. Biological effects of aminoguanidine: an update. Inflamm Res. 1999;48(10):509-15.

9. Dias AS, Porawski M, Alonso M, Marroni N, Collado PS, González-Gallego J. Quercetin decreases oxidative stress, NF-kappaB activation, and iNOS overexpression in liver of streptozotocin-induced diabetic rats. J Nutr. 2005;135(10):2299-304.

10. Degenhardt TP, Fu MX, Voss E, Reiff K, Neidlein R, Strein $\mathrm{K}$, et al. Aminoguanidine inhibits albuminuria, but not the formation of advanced glycation end-products in skin collagen of diabetic rats. Diabetes Res Clin Pract. 1999;43(2):81-9.

11. Llesuy SF, Milei J, Molina H, Boveris A, Milei S. Comparison of lipid peroxidation and myocardial damage induced by adriamycin and 4'-epiadriamycin in mice. Tumori. 1985;71(3):241-9.

12. Buege JA, Aust SD. Microsomal lipid peroxidation. Methods Enzymol. 1978;52:302-10.

13. Lowry OH, Rosebrough NJ, Farr AL, Randall RJ. Protein measurement with the Folin phenol reagent. J Biol Chem. 1951;193(1):265-75.

14. Giardino 1, Fard AK, Hatchell DL, Brownlee M. Aminoguanidine inhibits reactive oxygen species formation, lipid peroxidation, and oxidant-induced apoptosis. Diabetes. 1998;47(7):1114-20.

15. Kelly DJ, Gilbert RE, Cox AJ, Soulis T, Jerums G, Cooper ME. Aminoguanidine ameliorates overexpression of prosclerotic growth factors and collagen deposition in experimental diabetic nephropathy. J Am Soc Nephrol. 2001;12(10):2098-107.

16. Saler T, Cakmak G, Saglam ZA, Ataoglu E, Yesim Erdem T, Yenigun M. The assessment of pulmonary diffusing capacity in diabetes mellitus with regard to microalbuminuria. Intern Med. 2009;48(22):1939-43.

17. Goh SY, Cooper ME. Clinical review: The role of advanced glycation end products in progression and complications of diabetes. J Clin Endocrinol Metab. 2008;93(4):114352.

18. Popov D, Simionescu M. Alterations of lung structure in experimental diabetes, and diabetes associated with hyperlipidaemia in hamsters. Eur Respir J. 1997;10(8):1850-8.

19. Watanabe K, Senju S, Toyoshima H, Yoshida M. Thickness of the basement membrane of bronchial epithelial cells in lung diseases as determined by transbronchial biopsy. Respir Med. 1997;91(7):406-10.

20. Eren G, Cukurova Z, Hergunsel O, Demir G, Kucur M, Uslu E, et al. Protective effect of the nuclear factor kappa B inhibitor pyrrolidine dithiocarbamate in lung injury in rats with streptozotocin-induced diabetes. Respiration. 2010;79(5):402-10.

\section{Sobre os autores}

Fabio Cangeri Di Naso

Mestrando. Programa de Pós-Graduação em Ciências Biológicas, Universidade Federal do Rio Grande do Sul - UFRGS- Porto Alegre (RS) Brasil.

\section{Luiz Alberto Forgiarini Junior}

Doutorando. Programa de Pós-Graduação em Ciências Pneumológicas, Universidade Federal do Rio Grande do Sul - UFRGS - Porto Alegre (RS) Brasil.

\section{Luiz Felipe Forgiarini}

Acadêmico de Ciências Biológicas. Centro Universitário Metodista do Instituto Porto Alegre - IPA - Porto Alegre (RS) Brasil.

\section{Marilene Porawski}

Professora, Universidade Federal de Ciências da Saúde de Porto Alegre - UFCSPA - Porto Alegre (RS) Brasil.

\section{Alexandre Simões Dias}

Docente. Mestrado Profissional em Reabilitação e Inclusão, Centro Universitário Metodista do Instituto Porto Alegre - IPA - Porto Alegre (RS) Brasil.

\section{Norma Anair Possa Marroni}

Coordenadora do Laboratório de Hepatologia Experimental e Fisiologia, Universidade Federal do Rio Grande do Sul - UFRGS - Porto Alegre (RS); e Coordenadora do Laboratório de Estresse Oxidativo e Antioxidantes, Universidade Luterana do Brasil - ULBRA - Canoas (RS) Brasil. 